\title{
Charge Density Wave State of Monolayers in Graphite Intercalation Compounds
}

\author{
Andrei L. Tchougréeff \\ Karpov Institute of Physical Chemistry, Vorontsovo pole 10, Moscow, 103064, Russia \\ Received: November 7, 1995; In Final Form: May 14, $1996^{\otimes}$
}

\begin{abstract}
The electronic structure of a graphite monolayer with electron count corresponding to the graphite intercalation compound (GIC), described by the extended Hubbard Hamiltonian, is studied in the unrestricted HartreeFock approximation. We also interpret the data on observed STM images of graphite intercalation compounds. The well-known (and puzzling) pattern of graphite STM images with only three of the six atoms of each carbon hexagon visible is tentatively explained by the formation of a charge density wave (CDW) state of the surface graphite monolayer, which is an intrinsic feature of its electronic structure, rather than invoking wellknown attributions of the observed effect to structural differences between the sites and/or to interactions external to the monolayer. A tentative, purely electronic, explanation for the Moiré patterns is proposed as well.
\end{abstract}

\section{Introduction}

The surface of graphite and of its intercalation compounds (GIC) has been the subject of many scanning tunneling microscopy (STM) studies. ${ }^{1-4}$ In most STM images of graphite one observes only three of the six carbon atoms forming the well-known carbon hexagons of the graphite lattice or at least the tunnel currents differ significantly for the adjacent sites in the lattice. In the case of highly oriented pyrolytic graphite (HOPG) this feature conforms to its structure. The interactions between the layers make the carbon atoms of the surface layer nonequivalent. The A-type carbon atoms have an atom from another layer immediately under them, whereas the B-type atoms are located above the centers of the hexagons of the layer beneath. Atoms of one of these two types are believed to be more protruded on the STM images of graphite surfaces. ${ }^{1}$ This structural asymmetry, however, disappears when alkali metal atoms are intercalated between the graphite layers, yielding the stage-1 GICs of the general formula $\mathrm{MC}_{8}$, where $\mathrm{M}=\mathrm{Na}, \mathrm{K}$, $\mathrm{Rb}$, and Cs. The STM experiments on $\mathrm{GICs}^{5,6}$ were designed to prove that the observed STM images of neat HOPG arise from differences in electron density on the A- and B-sites, which in turn are driven by the structural differences of these sites in HOPG. Since in the GICs the structural difference between A- and B-sites in graphite monolayer disappears, the STM images of GICs predicted by the LDA calculation ${ }^{7,8}$ were not supposed to show the period of $2.46 \AA$, normally seen in the STM images of HOPG, corresponding to the above structural asymmetry. The outcome of the experiments, ${ }^{5,6}$ however, was in sharp contrast with these expectations. The STM images of stage-1 GIC $\mathrm{KC}_{8}$ still bear the features with the period of 2.46 $\AA$, as do the STM images of HOPG and of the graphite monolayer deposited on the $\operatorname{Pt}(111)$ surface. ${ }^{9}$ This structural pattern exists in addition to the superstructure with a period of $4.9 \AA$ (which matches the $\mathrm{K}-\mathrm{K}$ separation in $\mathrm{KC}_{8}$ ), despite the fact that the structural asymmetry between the A- and B-sites of the graphite monolayer is removed in GICs and in the monolayer deposited on the Pt surface.

It has been recently proposed ${ }^{10}$ that the observed STM images of HOPG, GICs, and the graphite monolayers deposited on the $\mathrm{Pt}(111)$ surface all manifesting the famous three-for-a-hexagon

${ }^{\otimes}$ Abstract published in Advance ACS Abstracts, July 15, 1996. pattern do so due to intrinsic features of the electronic structure of the graphite monolayers themselves rather than because of the interaction with the underlying layers or due to an action of any other external forces. It has been shown ${ }^{10,11}$ that the graphite monolayer can itself have an asymmetric electronic ground state with either charge or spin density waves (CDW or SDW). The existence of these states depends on specific values of parameters of electron-electron interactions in the layer. For realistic values of these parameters the CDW state was shown in ref 10 to have the lowest energy. However, the calculations in ref 10 have been performed for the case of the graphite monolayer with exactly one electron per carbon site rather than for a GIC. In the former case graphite is a gapless semiconductor, and its filled and empty bands touch each other at the points $\pm \mathbf{k}_{0}$ of the Brillouin zone (BZ) where $\mathbf{k}_{0}=(2 \pi / 3$, $-2 \pi / 3)$. This feature was considered a prerequisite for formation of unsymmetrical states and was a basis for the early explanation of the observed STM images of graphite by Tersoff. $^{12}$ It is almost generally accepted today that the threefor-a-hexagon pattern observed in the STM images of graphite monolayers in different situations is due to some electronic state with the nonuniform density distribution (CDW state). $5,6,10,12-14$ The current controversy is related to the origin of the state with a nonuniform charge distribution observed by the STM method. The main difference between the various explanations is the driving force of the nonuniform distribution. The authors ${ }^{10}$ suggest that the electron-electron interaction in the layer is itself this driving force, whereas other authors are inclined to ascribe the observed features to actions of some forces external to the layer. In the latter case the STM images of graphite and GICs have to be very sensitive to any variation of its close environment, which apparently does not happen: neither deposition on the Pt(111) surface nor intercalation changes the observed basic STM pattern (see above).

In this paper we consider the electronic structure of graphite monolayer in the case where some number of electrons is dumped from the alkaline atoms to the graphite $\pi$-band. With excessive electrons the monolayer is not a gapless semiconductor but a metal, ${ }^{15}$ and the reasoning ${ }^{12}$ does not apply to it. However, the equations describing the electronic structure of the monolayer in the Hartree-Fock approximation still have asymmetric solutions even for the average number of electrons per carbon 


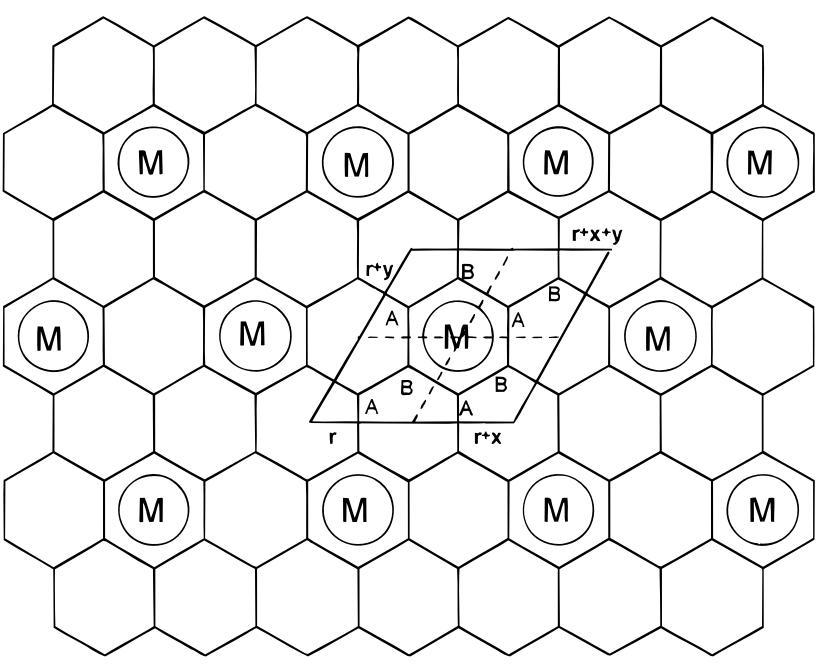

Figure 1. Structure of a graphite monolayer in the stage-1 GIC $\mathrm{MC}_{8}$. The enlarged $2 \times 2$ unit cell, which consists of four original unit cells, is shown. The A- and B-types of atoms are merely labels and have no relevance to the actual crystal structure.

site not much different from unity. In ref 14 it has been suggested that the Coulomb interaction with the intercalating metal cations is responsible for the charge localization in the layer. However, the calculation in ref 14 was done in such a way that the intersite electron hopping ultimately responsible for the smearing of electron density in the layer was not taken into account, and in that respect the effect of localization was overestimated. On the other hand, the Coulomb interaction between the electrons on the adjacent sites that played a key role in the electron localization in ref 10 was omitted in ref 14 . Moreover, it turns out that the most energetically favorable charge distribution in ref 14 corresponds to an STM image without the $2.46 \AA$ period. This most localized distribution has the periodicity of the underlying potassium cation layer and can account for the $4.9 \AA$ period only. The next less energetically favorable distribution had very weak features with a period of $2.46 \AA$, which also does not agree with experiment, ${ }^{5,6}$ where this structure is well pronounced. To summarize, the approach ${ }^{14}$ does not allow the superimposition of the two most important characteristic features of the STM image of the graphite monolayer in GIC-the 4.9 and $2.46 \AA$ periods-and does not permit them to be together in a single solution for the electronic structure of a monolayer. That is what we provide in this paper. In what follows we consider, first, the theoretical STM image as seen from the CDW state of the graphite monolayer with $n$ $=9 / 8$ with no external field; second, we describe the effect of perturbation induced by the metal cations on the $\pi$-electrons of the layer on the STM images of the GIC surface; and, third, we describe a tentative purely electronic origin of the Moiré patterns observed in the STM images of graphite.

\section{Extended Hubbard Model for Graphite Monolayer}

The extended Hubbard model Hamiltonian has been known for years in the area of conjugated $\pi$-systems and won considerable attention in recent years in the context of high- $T_{\mathrm{c}}$ superconductors. It is flexible enough and allows a ground state with a nonuniform charge distribution (the charge density wave state, CDW) when repulsion between electrons occupying adjacent sites is strong enough (see below and ref 10). We will use this model in the present paper in order to describe possible ground states of the graphite monolayers in GIC.

The structure of the graphite monolayer is shown in Figure 1. The unit cell contains two equivalent carbon atoms $\mathrm{A}$ and
B. (One has to note that the site labels A and B in our case are merely labels and have no structural relevance). Let $a_{\mathbf{r} \sigma}$ and $b_{\mathbf{r} \sigma}$ be the operators annihilating electrons with spin projection $\sigma= \pm 1 / 2$ on the A- and B-sites, respectively, in the rth unit cell. Using these operators, we can write the extended Hubbard Hamiltonian for the graphite monolayer:

$$
\begin{aligned}
& H=-\beta \sum_{\mathbf{r}, \sigma}\left(a_{\mathbf{r} \sigma}^{+} b_{\mathbf{r} \sigma}+b_{\mathbf{r} \sigma}^{+} a_{\mathbf{r}+\mathbf{y} \sigma}+\text { h.c. }\right)+ \\
& \gamma_{0} \sum_{\mathbf{r}}\left(a_{\mathbf{r} \alpha}^{+} a_{\mathbf{r} \alpha} a_{\mathbf{r} \beta}^{+}+b_{\mathbf{r} \alpha}^{+} b_{\mathbf{r} \alpha} b_{\mathbf{r} \beta}^{+} b_{\mathbf{r} \beta}\right)+\gamma_{1} \sum_{\mathbf{r}, \sigma, \tau}\left(a_{\mathbf{r} \sigma}^{+} a_{\mathbf{r} \sigma} b_{\mathbf{r} \tau}^{+} b_{\mathbf{r} \tau}+\right. \\
& \left.b_{\mathbf{r} \sigma}^{+} b_{\mathbf{r} \sigma} a_{\mathbf{r}+\mathbf{x} \tau}^{+} a_{\mathbf{r}+\mathbf{x} \tau}+b_{\mathbf{r} \sigma}^{+} b_{\mathbf{r} \sigma} a_{\mathbf{r}+\mathbf{y} \tau}^{+} a_{\mathbf{r}+\mathbf{y} \tau}\right)-3 \gamma_{1} \sum_{\mathbf{r}, \sigma}\left(a_{\mathbf{r} \sigma}^{+} a_{\mathbf{r} \sigma}+\right. \\
& \left.b_{\mathbf{r} \sigma}^{+} b_{\mathbf{r} \sigma}\right)
\end{aligned}
$$

The first term (proportional to $\beta$ ) describes electron transfer from A- and B-sites in the rth unit cell to adjacent sites both inside and outside the unit cell (h.c. = Hermitean conjugate). The second term (proportional to $\gamma_{0}$ ) is the Hubbard on-site electron repulsion term. The third term (proportional to $\gamma_{1}$ ) describes the repulsion between electrons occupying adjacent sites. The last term describes the attraction of electrons to the cores of the adjacent carbon atoms, and $\mathbf{x}$ and $\mathbf{y}$ are the unit cell vectors.

To find the ground state of a graphite monolayer described by the Hamiltonian eq 1, we use the equation of motion method combined with the Hartree-Fock approximation (see ref 10 and references therein). Within the Hartree-Fock approximation some products of three fermion operators occurring in the equation of motion method are replaced by expressions containing averages over the ground state. The general form of the averages is usually chosen on the basis of physical assumptions. Let us assume the averages to have the symmetry of the original lattice, so that the total magnetic moment of any unit cell is zero. That assumption leads us to the following form of the "on-site" averages:

$$
\left\langle a_{\mathbf{r} \sigma}^{+} a_{\mathbf{r} \sigma}\right\rangle=n / 2+\delta_{\sigma} ;\left\langle b_{\mathbf{r} \sigma}^{+} b_{\mathbf{r} \sigma}\right\rangle=n / 2-\delta_{\sigma}
$$

where the parameters $\delta_{\sigma}$ must be determined from selfconsistency conditions (see below). The averages of the electron-hopping operators of the form $\left\langle a_{\sigma}^{+} b_{\sigma}\right\rangle$ are set to $P_{\sigma}$ for all types of pairs of the adjacent sites, both intracell and intercell ones. To assure the conservation of the total spin projection of the system, the averages of the form $\left\langle c_{\sigma} c_{-\sigma}\right\rangle$ are set to zero. We also restrict ourselves in the present work by the spin symmetric states and do not consider the SDW state. With this additional condition we have

$$
\delta_{\alpha}=\delta_{\beta}=\delta ; \quad P_{\alpha}=P_{\beta}=P
$$

After the Hartree-Fock approximation is applied and the averages of eqs 2 and 3 are inserted, the equations of motion for the one-electron operators become

$$
\begin{gathered}
\mathrm{i} \partial a_{\mathbf{r} \sigma} / \partial t=-B\left(b_{\mathbf{r} \sigma}+b_{\mathbf{r}-\mathbf{x} \sigma}+b_{\mathbf{r}-\mathbf{y} \sigma}\right)+(\alpha-\Delta) a_{\mathbf{r} \sigma} \\
\mathrm{i} \partial b_{\mathbf{r} \sigma} / \partial t=-B\left(a_{\mathbf{r} \sigma}+a_{\mathbf{r}+\mathbf{x} \sigma}+a_{\mathbf{r}+\mathbf{y} \sigma}\right)+(\alpha+\Delta) b_{\mathbf{r} \sigma}
\end{gathered}
$$

where

$$
\begin{gathered}
\alpha=\gamma_{0} n / 2+3(n-1) \gamma_{1} \\
B=\beta+\gamma_{1} P \\
\Delta=\left(6 \gamma_{1}-\gamma_{0}\right) \delta
\end{gathered}
$$

In terms of the Fourier transforms (Bloch sums) of the site operators, 


$$
\begin{aligned}
& a_{\mathbf{k} \sigma}=\frac{1}{\sqrt{N}} \sum_{\mathbf{r}} \exp (-\mathbf{i k r}) a_{\mathbf{r} \sigma} \\
& b_{\mathbf{k} \sigma}=\frac{1}{\sqrt{N}} \sum_{\mathbf{r}} \exp (-\mathbf{i k r}) b_{\mathbf{r} \sigma}
\end{aligned}
$$

( $\mathbf{k}=\left(k_{x}, k_{y}\right)$ is the wave vector in the Brillouin zone (BZ); $N$ is the number of unit cells) the equations of motion take the form

$$
\begin{gathered}
\mathrm{i} \partial a_{\mathbf{k} \sigma} / \partial t=-B\left(1+\mathrm{e}^{i k_{x}}+\mathrm{e}^{i k_{y}}\right) b_{\mathbf{k} \sigma}+(\alpha-\Delta) a_{\mathbf{k} \sigma} \\
\mathrm{i} \partial b_{\mathbf{k} \sigma} / \partial t=-B\left(1+\mathrm{e}^{-i k_{x}}+\mathrm{e}^{-i k_{y}}\right) a_{\mathbf{k} \sigma}+(\alpha+\Delta) b_{\mathbf{k} \sigma}
\end{gathered}
$$

The solutions of these equations (Bloch states) can be written in the form

$$
\begin{gathered}
f_{\mathbf{k} \sigma}=x_{\mathbf{k}} a_{\mathbf{k} \sigma}+y_{\mathbf{k}} b_{\mathbf{k} \sigma} \\
g_{\mathbf{k} \sigma}=-y_{\mathbf{k}}^{*} a_{\mathbf{k} \sigma}+x_{\mathbf{k}}^{*} b_{\mathbf{k} \sigma} \\
\left|x_{\mathbf{k}}\right|^{2}+\left|y_{\mathbf{k}}\right|^{2}=1
\end{gathered}
$$

Corresponding dispersion laws (orbital energies) are

$$
\begin{gathered}
\epsilon_{\mathbf{k}}^{ \pm}=\alpha \pm R_{\mathbf{k}}, \quad R_{\mathbf{k}}=\sqrt{\Delta^{2}+B^{2} \epsilon_{0}^{2}(\mathbf{k})} \\
\epsilon_{0}{ }^{2}(\mathbf{k})=3+2 \cos k_{x}+2 \cos k_{y}+2 \cos \left(k_{x}-k_{y}\right)
\end{gathered}
$$

The coefficients $x_{\mathbf{k}}$ and $y_{\mathbf{k}}$ satisfy the conditions

$$
\left|x_{\mathbf{k}}\right|^{2}-\left|y_{\mathbf{k}}\right|^{2}=\Delta / R_{\mathbf{k}}, \quad x_{\mathbf{k}} y_{\mathbf{k}}^{*}=B\left(1+e^{-\mathrm{i} k_{x}}+e^{-\mathrm{i} k_{y}}\right) / R_{\mathbf{k}}
$$

The averages introduced above must be expressed as sums over the occupied states. We assume for the moment that the only effect of intercalation on the graphite monolayer is transfer of electrons from alkaline atoms to the $\pi$-bands of the layer. The filling of the states is then as follows: The Bloch $f$-states corresponding to the minus sign in the dispersion law are all occupied. This corresponds to the electron count of one electron per carbon atom. ${ }^{10}$ Additional electrons are placed in the Bloch g-states (corresponding to the plus sign in the dispersion law). In the vicinity of the $\pm \mathbf{k}_{0}$ points we introduce a new wave vector variable $\mathbf{q}=\mathbf{k}-\mathbf{k}_{0}$; then in the vicinity of the band edges the dispersion laws become

$$
\epsilon_{\mathbf{k}}^{ \pm}=\alpha \pm\left(\Delta^{2}+B^{2}\left(q_{x}^{2}-q_{x} q_{y}+q_{y}^{2}\right)\right)^{1 / 2}
$$

and expanding the square root, we get

$$
\epsilon_{\mathbf{k}}^{ \pm}=\alpha \pm \Delta \pm w\left(q_{x}^{2}-q_{x} q_{y}+q_{y}^{2}\right)
$$

where

$$
W=B^{2} / 2 \Delta
$$

Diagonalizing the quadratic form in $q$ 's we get

$$
\epsilon_{\mathbf{k}}^{ \pm}=\alpha \pm \Delta \pm W\left(3 / 2 q_{\xi}^{2}+1 / 2 q_{\eta}^{2}\right)
$$

with

$$
q_{\xi}=\left(q_{x}-q_{y}\right) / \sqrt{2} ; \quad q_{\eta}=\left(q_{x}+q_{y}\right) / \sqrt{2}
$$

In two dimensions the density of states is constant (provided the dispersion is quadratic in wave vector variables). According to ref 16 the effective mass entering the two-dimensional density of states is the geometrical average of the two masses, and we can write for the Fermi energy measured from the bottom of the $\mathrm{Hg}$-band

$$
\epsilon_{\mathrm{F}}=\sqrt{3} \pi W(n-1) / 4
$$

It is easy to check that each doubly occupied Bloch f-state contributes $2\left|x_{\mathbf{k}}\right|^{2} / N$ to the electron density on each A-site, $2\left|y_{\mathbf{k}}\right|^{2} / N$ to that on each B-site, and $2 x_{\mathbf{k}} y_{\mathbf{k}}^{* / N}$ to the bond order between the A- and B-sites. Each doubly occupied Bloch g-state contributes $2\left|y_{\mathbf{k}}\right|^{2} / N$ to the density on the A site, $2\left|x_{\mathbf{k}}\right|^{2} / N$ to that on the B-site, and $-2 x_{\mathbf{k}} y_{\mathbf{k}}^{*} / N$ to the bond order. Now one can easily see that

$$
\begin{gathered}
\delta=(1 / 2) \sum\left(\left|x_{\mathbf{k}}\right|^{2}-\left|y_{\mathbf{k}}\right|^{2}\right) \\
P=\sum x_{\mathbf{k}} y_{\mathbf{k}}^{*}
\end{gathered}
$$

where the summation is extended to the values of $\mathbf{k}$ for which $\epsilon_{\mathbf{k}}^{+} \geq \epsilon_{\mathrm{F}}$ (i.e. to the empty states). Equations 5, 10, and 16 form the system of self-consistency equations. This system always has a uniform state with $\delta=0$ as its solution. However, for the parameter values adopted in ref $10\left(\beta=2.4 \mathrm{eV}, \gamma_{0}=\right.$ $6 \mathrm{eV}, \gamma_{1}=2.5 \mathrm{eV}$ ) the CDW solution with the charge asymmetry between the A- and B-sites can be obtained also for the electron count corresponding to the complete transfer of electrons from potassium atoms to the layer $(n=9 / 8)$. We performed respective calculations and found that for the parameter set given above and for the complete electron transfer from the metal atoms to the layer the CDW state indeed appears and the value of the parameter of charge asymmetry $\delta$ is 0.16 ; $\Delta=1.47 \mathrm{eV} ; B=2.89 \mathrm{eV} ; W=3.02 \mathrm{eV}$; and $\epsilon_{\mathrm{F}}=0.51 \mathrm{eV}$.

\section{Ground State and STM Image of GIC}

In the previous section we obtained a CDW state for the graphite monolayer with $9 / 8 \pi$-electrons per carbon atom. Now we consider what effect this feature of the electronic structure of GIC could have on its observed STM image. According to ref 17, the observed STM image is proportional to $\rho\left(x, \epsilon_{\mathrm{F}}\right)$, the local density of states (LDOS) of the surface at the Fermi energy. In our case, the LDOS we are concerned with is that of a graphite monolayer with some electron count $1 \leq n \leq 9 / 8$. The general form for the LDOS is given by ${ }^{18}$

$$
\rho\left(x, \epsilon_{\mathrm{F}}\right)=2 / \pi \operatorname{Im} G\left(x, x, \epsilon_{\mathrm{F}}\right)
$$

where the one-electron Green's function is a formal solution of the respective eigenvalue problem:

$$
G(z)=(z I-F)^{-1}
$$

where $F$ is the self-consistent Fock operator. In the coordinateenergy representation it is given by

$$
G\left(x, x^{\prime}, z\right)=\sum_{v} \psi_{v}^{*}(x) \psi_{v}\left(x^{\prime}\right) /\left(z-\epsilon_{v}\right)
$$

where $\psi_{v}(x)$ is the $v$ th eigenstate of the eigenvalue problem with the Fock operator $F$ in the coordinate representation and $\epsilon_{v}$ is the corresponding orbital energy. One can easily check the following:

$$
\rho(x, E)=2 / \pi \operatorname{Im} G(x, x, E)=2 \sum_{v} \psi_{\nu}^{*}(x) \psi_{v}(x) \delta\left(E-\epsilon_{v}\right)
$$

where $E$ is a real energy at which the LDOS is to be found. In the site representation the coordinate $x$ on the surface can be 
replaced by an indication of the unit cell vector $\mathbf{r}$ and by specification (A or B) of the site in the unit cell.

For the graphite monolayer considered in the previous section the Fermi level is located in the upper band and the summation is extended to the Bloch states $g_{\mathbf{k}}$ with $\epsilon_{\mathbf{k}}^{+}=\epsilon_{\mathrm{F}}$, which are all located in the vicinity of the two characteristic minima of the band $\pm \mathbf{k}_{0}$. The Fermi surface is formed by two ellipses (one with the center at $\mathbf{k}_{0}$ another at $-\mathbf{k}_{0}$ ) defined by the equation

$$
\left(3 q_{\xi}^{2}+q_{\eta}^{2}\right)=\sqrt{3} \pi(n-1) / 2
$$

or parametrically

$$
\begin{aligned}
& q_{\xi}=a \cos \varphi ; \quad q_{\eta}=b \sin \varphi ; \quad b^{2}=\sqrt{3} \pi(n-1) / 2 ; \\
& a^{2}=b^{2} / 3
\end{aligned}
$$

so the summation (or integration) must be performed along these two ellipses in the BZ. From the explicit form of the Bloch g-states eq 8 we see that the LDOS of interest is $\mathbf{r}$ independent but depends on the site in the unit cell:

$$
\begin{aligned}
& \rho\left(A, \epsilon_{\mathrm{F}}\right)=2 \sum_{\mathbf{k}}\left|y_{\mathbf{k}}\right|^{2} \delta\left(\epsilon_{\mathrm{F}}-\epsilon_{\mathbf{k}}^{+}\right) \\
& \rho\left(B, \epsilon_{\mathrm{F}}\right)=2 \sum_{\mathbf{k}}\left|x_{\mathbf{k}}\right|^{2} \delta\left(\epsilon_{\mathrm{F}}-\epsilon_{\mathbf{k}}^{+}\right)
\end{aligned}
$$

(due to the $\delta$-functions, the summation here is effectively restricted to the states on the Fermi surface and the expression is multiplied by $N$ in order to work with the LDOS per unit cell). One can easily see that for the difference of the LDOS at the $\mathrm{B}$ and the $\mathrm{A}$ sites the following relation holds:

$$
\rho\left(B, \epsilon_{\mathrm{F}}\right)-\rho\left(A, \epsilon_{\mathrm{F}}\right)=2 \sum_{\mathbf{k}}\left(\left|x_{\mathbf{k}}\right|^{2}-\left|y_{\mathbf{k}}\right|^{2}\right) \delta\left(\epsilon_{\mathrm{F}}-\epsilon_{\mathbf{k}}^{+}\right)
$$

Substituting eq 10 for $\left(\left|x_{\mathbf{k}}\right|^{2}-\left|y_{\mathbf{k}}\right|^{2}\right)$, we get in the vicinity of the Fermi energy

$$
\left(\left|x_{\mathbf{k}}\right|^{2}-\left|y_{\mathbf{k}}\right|^{2}\right)=1 /\left(1+W\left(3 q_{\xi}^{2}+q_{\eta}^{2}\right) / 2 \Delta\right)
$$

On the Fermi surface the wave vector variables $q_{\xi}$ and $q_{\eta}$ satisfy eq 21 so that the above difference becomes a constant:

$$
\left(\left|x_{\mathbf{k}}\right|^{2}-\left|y_{\mathbf{k}}\right|^{2}\right)=1 /\left(1+\sqrt{3} \pi(n-1) B^{2} / 8 \Delta^{2}\right)
$$

This must be multiplied by 2 and by the ellipse length $L=$ $4 b \mathbf{E}(\sqrt{2 / 3})$, where $\mathbf{E}$ is the full elliptic integral of the second kind.

We can see now that the LDOS of the graphite monolayer with the electron count $n \geq 1$ is not symmetric with respect to the A- and B-sites in the unit cell provided the ground state of the layer is a CDW state driven by an electronic instability described above. According to Tersoff, ${ }^{17}$ this means that the tunnel current from such a surface must depend on the tip position above the latter and its STM images must manifest a pattern with the period $2.46 \AA$ (the famous three-for-a-hexagon pattern), in fair agreement with experiment. ${ }^{5,6}$ An important remark should be given here. It is mentioned in ref 14 that the experiments on the alternation of the bias voltage sign could be an important probe of the driving force of the CDW formation in the graphite monolayers since they allow probing of the LDOS of the surface both slightly above and slightly below the Fermi energy. It is absolutely true. However, it was stated in ref 14 that the variation of the bias voltage sign had to result in the change of the peak registry if the CDW state is driven by the intrinsic electron instability of the layer. This is not true, as one can easily see from a generalization of the above expression eq 23 for the LDOS's difference at the A- and B-sites. For any energy $E$ in the vicinity of the Fermi energy the LDOS's difference is given by

$$
\rho(B, E)-\rho(A, E)=16 b(E) \mathbf{E}(\sqrt{2 / 3}) /\left(1+\left(E-\epsilon_{\mathbf{k}_{0}}^{+}\right) / W\right)
$$

It is a slow varying function of energy (as it must be, by the way, for any metal) so that it is not going to abruptly change its sign (which would correspond to the change of the peak registry) when $E$ crosses the Fermi level, which is by no means chosen among other energy values. That is exactly what happens in experiment ${ }^{5,6}$ when the sign of the bias voltage is changed: the STM images of the $\mathrm{KC}_{8}$ surface have the same peak registry for either sign of the bias voltage, but in contrast to the statement of ref 14 it does not mean that the CDW is not driven by electron-electron interactions.

Finally we address the LDOS of a real stage-1 GIC. In the real compound the electronic structure of the surface graphite monolayer with $1 \leq n \leq 9 / 8$ is perturbed by the Coulomb field of the metal cations intercalated between the surface layer and the one beneath. The Coulomb interaction between the electrons in the layer and the metal cations gives an additional potential so that all the electronic states are shifted down in energy. However, the one-center attraction is renormalized by this interaction to a slightly different extent depending on whether the carbon atom at hand belongs to a six-membered ring just above a metal cation or it is an atom joining these rings (there are two of them per an enlarged unit cell of eight carbon atoms). The difference of these potentials gives the nontrivial part of the perturbation which is proportional to $V$. The electrons in the joining sites are effectively pushed up in energy by $V$. The perturbation operator takes the form

$$
H^{\prime}=V \sum_{\mathbf{r}, \sigma}^{\prime}\left(a_{\mathbf{r} \sigma}^{+} a_{\mathbf{r} \sigma}+b_{\mathbf{r}+\mathbf{x}+\mathbf{y} \sigma}^{+} b_{\mathbf{r}+\mathbf{x}+\mathbf{y} \sigma}\right)
$$

where the summation is extended to the unit cell vectors $\mathbf{r}$, both components of which are even integers. These vectors enumerate the unit cells of the enlarged $2 \times 2$ lattice of the perturbed problem. To study the effect of the perturbation, it is more convenient to use the representation of folded bands corresponding to the enlarged unit cell (for more details see ref 19). In this representation instead of the two bands and wave vectors in the Brillouin zone (BZ) we have eight bands and wave vectors confined to the reduced Brillouin zone (RBZ). Any wave vector of the BZ can be presented uniquely in one of the forms $\mathbf{k}^{(0)}=$ $\left(k_{x}, k_{y}\right), \mathbf{k}^{(1)}=\left(k_{x}-\pi, k_{y}\right), \mathbf{k}^{(2)}=\left(k_{x}, k_{y}-\pi\right)$, and $\mathbf{k}^{(3)}=\left(k_{x}\right.$ $\left.-\pi, k_{y}-\pi\right)$ for each $\mathbf{k}=\mathbf{k}^{(0)}=\left(k_{x}, k_{y}\right)$, provided the wave vector $\mathbf{k}$ is confined to the RBZ subject to the conditions $0 \leq$ $k_{x}, k_{y} \leq \pi$. In this representation the $\mathrm{f}$ and $\mathrm{g}$ Bloch states of the above CDW solution are labeled by a wave vector $\mathbf{k} \in \mathrm{RBZ}$ and by a new quantum number $i(=0,1,2,3)$ as follows:

$$
f_{i \mathbf{k}}=f_{\mathbf{k}^{(i)}}, \quad g_{i \mathbf{k}}=g_{\mathbf{k}^{(i)}}
$$

The Bloch f-states are filled for all $\mathbf{k}$ and $i$. The Fermi level $\epsilon_{\mathrm{F}}$ crosses the g-bands for $i=1,2$. The characteristic points $\pm \mathbf{k}_{0}$ of the BZ acquire, respectively, the following forms:

$$
\mathbf{k}_{0}=\mathbf{K}_{1}^{(1)} ; \quad-\mathbf{k}_{0}=\mathbf{K}_{2}^{(2)}
$$

where the wave vectors $\mathbf{K}_{1}=(\pi / 3,2 \pi / 3), \mathbf{K}_{2}=(2 \pi / 3, \pi / 3)$ belong to the RBZ. The Fermi surface consists of two ellipses, $\mathbf{K}_{i}+\mathbf{q}, i=1,2$, with the wave vector $\mathbf{q}$ subject to the above condition in eq 21 with the understanding that each of the two segments of the Fermi surface appears in the respective band $\mathrm{g}_{\mathbf{i k}}$. 
For each $\mathbf{k} \in \mathrm{RBZ}$ the perturbation $H^{\prime}$ (eq 26) couples the Bloch states with the wave vectors $\mathbf{k}^{(i)}$. It also shifts all the states by $V$; however, it does not couple the Bloch states $\mathrm{f}_{i \mathbf{k}}$ and $\mathrm{g}_{i \mathbf{k}}$ for the same $i$. One can easily check that the diagonal part of the perturbation operator $H^{\prime}$ equals $V$ for all $\mathbf{k}$; that is, it is the uniform shift of all levels by $V$. Hereinafter we omit the diagonal part of the perturbation operator $H^{\prime}$ and consider only the nontrivial (off-diagonal) part of the perturbation.

Now we consider the LDOS of a perturbed monolayer. As we mentioned above, $G(z)=(z I-F)^{-1}$. According to the general formula the Green's function for the perturbed Hamiltonian (or Fockian), $H=H_{0}+H^{\prime}\left(F=F_{0}+H^{\prime}\right)$, is approximately given in terms of the Green's function $G_{0}=(z I$ $\left.-F_{0}\right)^{-1}$ for the unperturbed problem: ${ }^{18}$

$$
G(z)=\left(z I-F_{0}-H^{\prime}\right)^{-1} \simeq G_{0}(z)+G_{0}(z) H^{\prime} G_{0}(z)
$$

The imaginary part of first term in the above approximate expression for the Green's function gives the LDOS of the unperturbed monolayer, and that of the term quadratic in $G_{0}$ gives a correction $\delta \rho(x, E)$ to it. Using the general formula eq 19, we have

$$
G_{0}(z)=\sum_{i, \mathbf{k}}\left\{\left|f_{i \mathbf{k}}\right\rangle\left\langle f_{i \mathbf{k}}\left|/\left(z-\epsilon_{i_{\mathbf{k}}}^{-}\right)+\right| g_{i \mathbf{k}}\right\rangle\left\langle g_{i \mathbf{k}}\right| /\left(z-\epsilon_{i \mathbf{k}}^{+}\right)\right\}
$$

Then we have

$$
\begin{gathered}
\operatorname{Im} G_{0}\left(\epsilon_{\mathrm{F}}\right)=-\pi \sum_{i, \mathbf{k}}\left|g_{i \mathbf{k}}\right\rangle\left\langle g_{i \mathbf{k}}\right| \delta\left(\epsilon_{\mathrm{F}}-\epsilon_{i \mathbf{k}}^{+}\right) \\
\operatorname{Re} G_{0}\left(\epsilon_{\mathrm{F}}\right)=\sum_{i, \mathbf{k}}\left\{\left|f_{i \mathbf{k}}\right\rangle\left\langle f_{i \mathbf{k}}\left|/\left(\epsilon_{\mathrm{F}}-\epsilon_{i \mathbf{k}}^{-}\right)+\right| g_{i \mathbf{k}}\right\rangle\left\langle g_{i \mathbf{k}}\right| /\left(\epsilon_{\mathrm{F}}-\epsilon_{i \mathbf{k}}^{+}\right)\right\}
\end{gathered}
$$

Due to the $\delta$-functions, the sum over $\mathbf{k}$ in the formula for $\mathrm{Im}$ $G_{0}$ is restricted to the values $\mathbf{K}_{i}+\mathbf{q}$, where $\mathbf{q}$ is confined to the ellipse defined by eq 21 and $i=1,2$.

$$
\begin{gathered}
\delta \rho\left(x, \epsilon_{\mathrm{F}}\right)=\left\langle x\left|\operatorname{Im} G_{0}\left(\epsilon_{\mathrm{F}}\right) H^{\prime} G_{0}\left(\epsilon_{\mathrm{F}}\right)\right| x\right\rangle=-\pi \sum_{\mathbf{q}} \sum_{i=1,2} \sum_{j \neq i} \delta \\
\left(\epsilon_{\mathrm{F}}-\epsilon_{i \mathbf{K}_{i}+\mathbf{q}}^{+}\right)\left\{\left\langle x \mid g_{i \mathbf{K}_{i}+\mathbf{q}}\right\rangle\left\langle g_{i \mathbf{K}_{i}+\mathbf{q}}\left|H^{\prime}\right| f_{j \mathbf{K}_{i}+\mathbf{q}}\right\rangle\left\langle f_{j \mathbf{K}_{i}+\mathbf{q}} \mid x\right\rangle /\left(\epsilon_{\mathrm{F}}-\right.\right. \\
\epsilon_{j}^{-} \mathbf{K}_{i}+\mathbf{q} \\
)+\left\langle x \mid g_{i \mathbf{K}_{i}+\mathbf{q}}\right\rangle\left\langle g_{i \mathbf{K}_{i}+\mathbf{q}}\left|H^{\prime}\right| g_{j \mathbf{K}_{i}+\mathbf{q}}\right\rangle\left\langle g_{j \mathbf{K}_{i}+\mathbf{q}} \mid x\right\rangle /\left(\epsilon_{\mathrm{F}}-\right. \\
\left.\epsilon_{j \mathbf{K}_{i+\mathbf{q}}}^{+}\right)+\left\langle x \mid f_{j \mathbf{K}_{i}+\mathbf{q}}\right\rangle\left\langle f_{j \mathbf{K}_{i}+\mathbf{q}}\left|H^{\prime}\right| g_{i \mathbf{K}_{i}+\mathbf{q}}\right\rangle\left\langle g_{i \mathbf{K}_{i}+\mathbf{q}} \mid x\right\rangle /\left(\epsilon_{\mathrm{F}}-\epsilon_{j \mathbf{K}_{i}+\mathbf{q}}\right)+ \\
\left.\left\langle x \mid g_{j \mathbf{K}_{i}+\mathbf{q}}\right\rangle\left\langle g_{j \mathbf{K}_{i}+\mathbf{q}}\left|H^{\prime}\right| g_{i \mathbf{K}_{i}+\mathbf{q}}\right\rangle\left\langle g_{i \mathbf{K}_{i}+\mathbf{q}}\right\rangle\left\langle g_{i \mathbf{K}_{i}+\mathbf{q}} \mid x\right\rangle /\left(\epsilon_{\mathrm{F}}-\epsilon_{j \mathbf{K}_{i+\mathbf{q}}}^{+}\right)\right\}
\end{gathered}
$$

To evaluate the latter expression, we assume, first, that the $\mathbf{q}$ dependent orbital energies in the denominators are only weakly different from their value at $\mathbf{q}=0$ and can be replaced by the latter. One can easily check that for any $j \neq i$ the orbital energies are given by

$$
\epsilon_{j \mathbf{K}_{i}}^{ \pm}=\alpha \pm \sqrt{\Delta^{2}+4 B^{2}}
$$

For the purposes of estimating we also replace $\epsilon_{\mathrm{F}}$ in the denominators by $\epsilon_{\mathbf{k}_{0}}^{+}=\alpha+\Delta$ and obtain

$$
\left(\epsilon_{\mathrm{F}}-\epsilon_{j \mathbf{K}_{i}}^{ \pm}\right)=\Delta \mp \sqrt{\Delta^{2}+4 B^{2}}
$$

To evaluate the numerator, we replace all the matrix elements by their values in the respective points $\mathbf{K}_{i}^{(j)}$ assuming again that the effect of $\mathbf{q}$ is weak when the ellipse is not large and replace the summation over $\mathbf{q}$ by multiplying the expression under the summation sign by the length of the ellipse $L$.
In the perturbed system only the quarter of the original unit cell vectors corresponding to the cells of the enlarged $2 \times 2$ lattice can be used. The enlarged unit cell contains, in turn, the A- and B-sites with the lattice vectors $\mathbf{r}+\mathbf{t}$, where $\mathbf{t}$ takes four values: $\mathbf{0}, \mathbf{x}, \mathbf{y}$, and $\mathbf{x}+\mathbf{y}$. In the representation of the 2 $\times 2$ lattice sites the states $|x\rangle$ have the form $|\mathbf{r}, \mathbf{t}, A\rangle,|\mathbf{r}, \mathbf{t}, B\rangle$. One can check that the LDOS in the perturbed system is $\mathbf{r}$ independent. It is also easy to establish that, for $i=1,2, g_{i \mathbf{K}_{i}}$ $=b_{\mathbf{K}_{i}^{(i)}}$, or, in other words, $y_{\mathbf{K}_{i}^{(i)}}=y_{ \pm \mathbf{k}_{0}}=0 ; x_{K_{i}^{(i)}}=x_{ \pm \mathbf{k}_{0}}=1$. For that reason $\delta \rho\left(A, \mathbf{t}, \epsilon_{\mathrm{F}}\right)=0$ for any position $\mathbf{t}$ in the enlarged unit cell. The LDOS on the A-sites in the perturbed system does not differ from that in the unperturbed system. For the B-sites we have

$$
\begin{aligned}
& \delta \rho\left(\mathbf{t}, B, \epsilon_{\mathrm{F}}\right)= \\
& \quad-\pi L V \delta / B^{2} \sum_{i=1,2} \sum_{j \neq i} \exp \left(i\left(\mathbf{K}_{i}^{(i)}-\mathbf{K}_{i}^{(j)}\right) \mathbf{t}\right)(-1)^{\left(\delta_{j, 1}+\delta_{j, 2}\right)}
\end{aligned}
$$

Calculating the above sum for four values of $\mathbf{t}$ labeling different $\mathrm{B}$-sites in the enlarged unit cell we get the following:

$$
\begin{aligned}
& \delta \rho\left(\mathbf{0}, B, \epsilon_{\mathrm{F}}\right)=\delta \rho\left(\mathbf{x}, B, \epsilon_{\mathrm{F}}\right)=\delta \rho\left(\mathbf{y}, B, \epsilon_{\mathrm{F}}\right)= \\
&-2 \pi V \delta L / B^{2}=-\pi V / W \\
& \delta \rho\left(\mathbf{x}+\mathbf{y}, B, \epsilon_{\mathrm{F}}\right)=6 \pi V \delta L / B^{2}=3 \pi V / W
\end{aligned}
$$

We see that the resulting LDOS $\rho+\delta \rho$ retains the characteristic feature of the $2.46 \AA$ period, which is fixed by the unperturbed LDOS eqs 22 and 23. At the same time even within extremely simplified approximations that we adopted when evaluating the correction $\delta \rho$ to the LDOS it has a lower symmetry and depends on the position $\mathbf{t}$ in the enlarged unit cell. According to our result, the STM image of the enlarged unit cell must consist of four bright spots corresponding to the four B-sites in it. One of these spots must be brighter than the other three provided the sign of the perturbation parameter $V$ is positive. Thus the total LDOS has the periodicity of the $2 \times 2$ lattice. This all fairly corresponds to the observed STM image of the GIC surface. ${ }^{5,6}$ The periodicity of the observed STM image is that of the $2 \times 2$ lattice $(4.9 \AA)$. For any unit cell of the surface four bright spots are observed. One of them is the brightest p-site of refs 5 and 6 corresponding in our notation to the $\mathbf{x}+$ $\mathbf{y}$ B-site; the other three (two $\mathrm{p}^{\prime}$ - and one $\mathrm{p}^{\prime \prime}$-sites of refs 5, 6) are the sites of lower LDOS and of the lower brightness.

Another feature that could be observed as well in a tunnel experiment on GIC is the gap well below the Fermi energy. There are no states in the forbidden band of the width $\Delta$ below the bottom of the g-band. With the parameters accepted in ref 10 our approach predicts a gap of $1.47 \mathrm{eV}$ when the bias voltage $V_{\text {bias }}=\epsilon_{\mathrm{F}} / e$ grows stronger than $510 \mathrm{mV}$. However, the parameters of the electronic structure like the charge assymmetry parameter $\delta$, the Fermi energy $\epsilon_{\mathrm{F}}$, and the gap $\Delta$ determined by the Hartree-Fock approach are very sensitive to the parameters of the Hamiltonian. If we fix $\beta$ and $\gamma_{0}$ at their values adopted above and scan $\gamma_{1}$, we see that the CDW state disappears for the values of $\gamma_{1}$ of $2.4 \mathrm{eV}$ and smaller. Just above the threshold of the CDW state formation $\left(\gamma_{1}=2.41 \mathrm{eV}\right)$ we find the following parameters of the electronic structure for the $n=9 / 8$ filling: $\delta=0.08 ; \Delta=0.72 \mathrm{eV} ; B=2.98 \mathrm{eV} ; W=$ $6.13 \mathrm{eV}$; and $\epsilon_{\mathrm{F}}=1.04 \mathrm{eV}$. These figures correspond to the gap of $0.72 \mathrm{eV}$ at the bias voltage stronger than $1000 \mathrm{mV}$. From these data we can conclude that in a scanning tunnel spectroscopy (STS) experiment on a stage-1 GIC like $\mathrm{KC}_{8}$ one could expect a gap of the width 0.5 to $1.5 \mathrm{eV}$ somewhere 0.5 to 1.5 $\mathrm{eV}$ below the Fermi energy. It is difficult to say more from 
the purely theoretical point of view since the parameters of the Hamiltonian are not known with sufficient precision. On the other hand, according to ref 5, the $\mathrm{STM}$ image of $\mathrm{KC}_{8}$ does not change up to the bias voltage of $\pm 750 \mathrm{mV}$, thus indicating the lower limit for $\epsilon_{\mathrm{F}} \geq 0.75 \mathrm{eV}$.

The CDW state of GIC manifests itself not only in the tunnel experiments but also in some others. For example, the $\mathrm{C} 1 \mathrm{~s}-$ core excitation shows an additional absorption $0.7 \mathrm{eV}$ above the absorption edge. ${ }^{20}$ The ionization potential of a core electron is apparently sensitive to the population of the $\pi$-orbital on the same site. In the CDW state two types of core states with the energy shift of $2 \delta \Gamma$ exist. (Here $\Gamma$ is the Coulomb interaction between the core electron and the $\pi$-electron.) Our calculation on $\mathrm{KC}_{8}$ reproduces the observed splitting with $\Gamma \approx 5 \mathrm{eV}$, which seems to be a reasonable estimate. ${ }^{21}$ On the other hand, the calculation with the same parameter set, but with $n=7 / 6$, which corresponds to the $\mathrm{LiC}_{6} \mathrm{GIC}$, does not give any $\mathrm{CDW}$ solution, but only a uniform state with $\delta=0$. Incidentally the $\mathrm{C} 1 \mathrm{~s}-$ core absorption spectrum of $\mathrm{LiC}_{6}$ has only one peak at the ionization threshold. ${ }^{20}$

\section{Moiré Patterns and Fermi Surface Nesting}

The formation of the low-symmetry states driven by intrinsic interactions in an extended system is usually attributed to a specific property of the related noninteracting model that is called "Fermi surface nesting". When the nesting takes place for any wave vector $\mathbf{k}$ on the Fermi surface and for some wave vector $\mathbf{Q}$ called the "nesting vector", the following holds:

$$
\epsilon_{\mathbf{k}+\mathbf{Q}}=\epsilon_{\mathbf{k}}
$$

It is widely believed (see, for example, ref 14 and references therein) that the low-symmetry states with the superstructure wave vector $\mathbf{Q}$ appear only when the nesting occurs and vice versa. This is not, however, completely true. The nesting criterion for the formation of the low-symmetry states is rather a more useful hint than an exact theorem. Even for the systems with nesting the low-symmetry states do not appear if the interaction is weaker than the critical one, and this critical value depends on dimensionality of the system and details of its structure. On the other hand, the low-symmetry states may occur without nesting, as we have seen in the present paper (see also ref 10). In effect, there is no nesting that could lead to the formation of the CDW state with the period $2.46 \AA$. Nevertheless, as we show numerically in the present paper, for the realistic set of parameters of the extended Hubbard Hamiltonian the CDW state appears as a solution of the selfconsistency equations eqs 5, 10, and 16 for the graphite monolayer with electron count $n=9 / 8$ per carbon atom. This CDW state exists despite the fact that the nesting criterion apparently is not fulfilled for the graphite monolayer.

One can easily check, however, that in our problem there is a nontrivial wave vector for which the nesting condition is fulfilled. As we already mentioned, the Fermi surface of the graphite monolayer with $n \geq 1$ in the CDW state consists of two segments: two ellipses encircling respectively the points $\mathbf{k}_{0}$ and $-\mathbf{k}_{0}$ in the BZ. The two segments of the Fermi surface are connected by the nesting vector $\mathbf{Q}=2 \mathbf{k}_{0}$. For any wave vector $\mathbf{q}$ in the vicinity of the point $\mathbf{k}_{0}$ in the $\mathrm{BZ}$ we have

$$
\epsilon_{\mathbf{k}_{0}+\mathbf{q}}^{+}=\epsilon_{\mathbf{k}_{0}-\mathbf{Q}+\mathbf{q}}^{+}=\epsilon_{-\mathbf{k}_{0}+\mathbf{q}}^{+}
$$

which is, by definition, the nesting condition for the two segments of the Fermi surface. According to ref 22, the interaction coupling the Bloch states in the vicinity of the two segments of the Fermi surface may result in a low-symmetry state with the periodicity corresponding to the same wave vector Q. To estimate the possible effect of nesting on the observed STM images of GIC surface we apply again the Green's function method. According to the general formula, the Green's function for the perturbed Hamiltonian $H=H_{0}+H^{\prime}$, where the perturbation $H^{\prime}$ is an electron-electron interaction,

$$
H^{\prime}=\sum_{\mu \nu \lambda \kappa}(\mu \nu \mid \lambda \kappa) \psi_{\mu}^{+} \psi_{\nu} \psi_{\lambda}^{+} \psi_{\kappa}
$$

treated in a self-consistent manner is given in terms of the Green's function $G_{0}$ for the unperturbed problem and the socalled self-energy part $\sum(z)$ by the following expression:

$$
G^{-1}(z)=G_{0}^{-1}(z)-\Sigma(z)=\left(z I-F_{0}-\Sigma(z)\right)
$$

or

$$
G(z)=\left(z I-F_{0}-\Sigma(z)\right)^{-1}
$$

where the self-energy part

$$
\begin{gathered}
\Sigma_{\mu \nu}(z)=\sum_{\lambda, \kappa}[(\mu \nu \mid \lambda \kappa)-(\mu \kappa \mid \lambda \nu)] P_{\kappa \lambda} \\
P_{\kappa \lambda}=1 / 2 \pi i \int_{-\infty}^{\infty} \mathrm{d} E G_{\kappa \lambda}(E)
\end{gathered}
$$

is itself a functional of the Green's function to be found. The only difference of eq 39 from eq 29 is that the one-particle operator $\Sigma$ is itself $G$ dependent and eqs 39 or 40 and 41 form a system of equations for $G$ that have to be resolved selfconsistently. We, however, are not going to do it here, and we shall only estimate the correction $\delta \rho$ to the LDOS using eq 29 with $\Sigma(z)$ inserted instead of $H^{\prime}$ in the assumption that a nontrivial solution of the above system exists.

For our purposes we can rewrite the unperturbed Green's function eq 30 as follows:

$$
\begin{aligned}
& G_{0}(z)=\sum_{\mathbf{q}}\left\{\left|g_{\mathbf{k}_{0}+\mathbf{q}}\right\rangle\left\langle g_{\mathbf{k}_{0}+\mathbf{q}}\right| /\left(z-\epsilon_{\mathbf{k}_{0}+\mathbf{q}}^{+}\right)+\right. \\
&\left.\left|g_{-\mathbf{k}_{0}+\mathbf{q}}\right\rangle\left\langle g_{-\mathbf{k}_{0}+\mathbf{q}}\right| /\left(z-\epsilon_{-\mathbf{k}_{0}+\mathbf{q}}^{+}\right)\right\}
\end{aligned}
$$

since only the Bloch g-states in the vicinity of the Fermi surface are involved. The Green's function eq 42 can be presented as a sum of $2 \times 2$ diagonal matrices $G_{0}(\mathbf{q}, z)$ for each value of $\mathbf{q}$. The interaction we are concerned with is the Coulomb interaction between electrons in the vicinity of the two respective segments of the Fermi surface in the reciprocal (k-) space. The corresponding perturbation operator reads

$$
\begin{aligned}
H^{\prime}=\sum_{\mathbf{q}, \mathbf{p}, \sigma, \tau}\left(\mathbf{k}_{0}+\mathbf{q},-\mathbf{k}_{0}+\mathbf{q} \mid-\mathbf{k}_{0}+\mathbf{p}, \mathbf{k}_{0}+\right. \\
\mathbf{p}) g_{\mathbf{k}_{0}+\mathbf{q} \sigma}^{+} g_{-\mathbf{k}_{0}+\mathbf{p} \tau}^{+} g_{\mathbf{k}_{0}+\mathbf{p} \tau}
\end{aligned}
$$

and the corresponding self-energy acquires the form of a sum over $\mathbf{q}$ of $2 \times 2$ matrices as well:

$$
\begin{gathered}
\Sigma(z)=\sum_{\mathbf{q}} \Sigma(\mathbf{q}, z), \Sigma(\mathbf{q}, z)=\sum_{\mathbf{p}, \tau}\left|g_{\mathbf{k}_{0}+\mathbf{q}}\right\rangle\left\langle g_{-\mathbf{k}_{0}+\mathbf{q}}\right|\left(\mathbf{k}_{0}+\mathbf{q}\right. \\
\left.-\mathbf{k}_{0}+\mathbf{q} \mid-\mathbf{k}_{0}+\mathbf{p}, \mathbf{k}_{0}+\mathbf{p}\right) P_{\mathbf{p} \tau}^{+-} \\
P_{\mathbf{p} \tau}^{+-}=\left\langle g_{-\mathbf{k}_{0}+\mathbf{p} \tau}^{+} g_{\mathbf{k}_{0}+\mathbf{p} \tau}\right\rangle
\end{gathered}
$$

The off-diagonal matrix elements of the density matrix $P_{\mathbf{p} \tau}^{+-}$ are related to the off-diagonal elements of the $2 \times 2$ matrices of the $\mathbf{p}$ components of the exact Green's function eq 40, which are called "anomalous averages" in this context. ${ }^{22}$ Using the 
approximate form for the Bloch $g$-states in the vicinity of the $\pm \mathbf{k}_{0}$ points and the symmetry-conditioned independence of the anomalous averages on $\mathbf{p}$ and $\tau$, we easily get for the $\mathbf{q}$ components of the self-energy

$$
\begin{gathered}
\Sigma(\mathbf{q}, z)=\left|g_{\mathbf{k}_{0}+\mathbf{q}}\right\rangle\left\langle g_{-\mathbf{k}_{0}+\mathbf{q}}\right| \Xi+\text { h.c. } \\
\Xi=2 \gamma_{0} / N \sum_{\mathbf{p}, \tau} P_{\mathbf{p} \tau}^{+-}
\end{gathered}
$$

The quantity $\Xi$ is an order parameter ${ }^{22}$ nonvanishing in the lowsymmetry state with the nesting vector $\mathbf{Q}$. Finally we get the LDOS correction coming from the ordering with the vector $\mathbf{Q}$ using eqs 29-31:

$$
\begin{gathered}
G(z) \simeq G_{0}(z)+G_{0}(z) \Sigma G_{0}(z) \\
\operatorname{Im} G_{0}\left(\epsilon_{\mathrm{F}}\right)=-\pi \sum_{\mathbf{k}}\left|g_{\mathbf{k}}\right\rangle\left\langle g_{\mathbf{k}}\right| \delta\left(\epsilon_{\mathrm{F}}-\epsilon_{\mathbf{k}}^{+}\right) \\
\operatorname{Re} G_{0}\left(\epsilon_{\mathrm{F}}\right)=\sum_{\mathbf{k}}\left|g_{\mathbf{k}}\right\rangle\left\langle g_{\mathbf{k}}\right| /\left(\epsilon_{\mathrm{F}}-\epsilon_{\mathbf{k}}^{+}\right)
\end{gathered}
$$

Due to the $\delta$-functions the sum over $\mathbf{k}$ in the formula for $\operatorname{Im} G_{0}$ is restricted to the values $\pm \mathbf{k}_{0}+\mathbf{q}$, where $\mathbf{q}$ is confined to the ellipse defined by eq 21 . For the LDOS correction we have

$$
\begin{gathered}
\delta \rho\left(x, \epsilon_{\mathrm{F}}\right)=\left\langle x\left|\operatorname{Im}\left\{G_{0}\left(\epsilon_{\mathrm{F}}\right) \Sigma G_{0}\left(\epsilon_{\mathrm{F}}\right)\right\}\right| x\right\rangle= \\
-\pi \sum_{\mathbf{q}} \delta\left(\epsilon_{\mathrm{F}}-\epsilon_{\mathbf{k}_{0}+\mathbf{q}}^{+}\right)\left\{\left\langle x \mid g_{\mathbf{k}_{0}+\mathbf{q}}\right\rangle\left\langle g_{\mathbf{k}_{0}+\mathbf{q}}\left|\sum\right| g_{-\mathbf{k}_{0}+\mathbf{q}}\right\rangle\left\langle g_{-\mathbf{k}_{0}+\mathbf{q}} \mid x\right\rangle /\right. \\
\left(\epsilon_{\mathrm{F}}-\epsilon_{\mathbf{k}_{0}+\mathbf{q}}^{+}\right)+\left\langle x \mid g_{-\mathbf{k}_{0}+\mathbf{q}}\right\rangle\left\langle g_{-\mathbf{k}_{0}+\mathbf{q}}\left|\sum\right| g_{\mathbf{k}_{0}+\mathbf{q}}\right\rangle\left\langle g_{\mathbf{k}_{0}+\mathbf{q}} \mid x\right\rangle / \\
\left.\left(\epsilon_{\mathrm{F}}-\epsilon_{\mathrm{F}}-\epsilon_{\mathbf{k}_{0}+\mathbf{q}}^{+}\right)\right\} \\
\delta \varrho\left(\mathbf{r}, B, \epsilon_{F}\right) \propto \operatorname{Re} \Xi \exp (i \mathbf{Q r})
\end{gathered}
$$

We finally see that in the low-symmetry state with the order parameter $\Xi$ the LDOS correction for the B-sites has the wave vector $\mathbf{Q}$, and thus the STM image must have a superstructure with the same wave vector. The nesting vector $\mathbf{Q}=2 \mathbf{k}_{0}$ results in the 3-fold lattice parameter of the observed STM image. Combined with the cell doubling due to the interactions with the metal cations in the stage-1 GIC this results in the lattice constant 6 times as large as the original constant of $2.46 \AA$. The corresponding characteristic length amounts to about 15 $\AA$, which fairly corresponds to the lower limit (about $17 \AA$ ) of the Moiré superstructures observed experimentally. ${ }^{23}$ We do not mean to say that the Moiré patterns appear exclusively due to the ordered states with the $\mathbf{Q}=2 \mathbf{k}_{0}$ nesting, but we would like to attract attention to such a possibility. Of course, the tentative $\mathbf{Q}=2 \mathbf{k}_{0}$ nesting (if any) must manifest itself in a variety of ways. One of them must be a depletion of the DOS at the Fermi level due to formation of the ordered state, which is now tentatively detected by the tunnel experiments as the Moiré pattern.

\section{Discussion}

In the present paper we substantiated numerically the possibility of formation of the electronically driven CDW state in a graphite monolayer with an electron count corresponding to the stage-1 GIC and considered theoretically the STM images of the latter. The nonuniform distribution of the electron density in the graphite monolayer also has its consequences for the LDOS at the Fermi energy and thus for the observed STM images of GICs. We derived explicitly the LDOS of the monolayer in three cases. First, we considered the LDOS of the monolayer with $n=9 / 8$ but without external field. This LDOS manifests the most important feature characteristic for the all STM images of graphite observed in various situations: the $2.46 \AA$ periodicity or the three-for-a-hexagon pattern. In our approximation the LDOS is largely concentrated on the B-sites, and thus they look to be more protruded on the STM images. Moreover, the LDOS obtained in our paper has an important property as a function of energy. The LDOS difference between the B- and A-sites that corresponds to the registry of the bright and dark spots on the STM image does not change its sign when the energy at which the LDOS is probed switches from a value slightly above the Fermi energy to that slightly below it. According to ref 14, the switching of the polarity of the bias voltage had to result in change of the peak registry if the CDW state of the monolayer is intrinsically driven. One can see from eq 25 that it is not true since the sign of the LDOS difference does not depend on the probe energy (at least in the vicinity of the Fermi energy), and thus the peak registry need not change when the bias voltage changes its polarity even in the case of the intrinsically driven CDW.

The effect of the external field induced by the metal cations on the LDOS of the monolayer is in remarkable agreement with the experiments. ${ }^{5,6} \mathrm{We}$ note that under the influence of the cations' field the LDOS is asymmetrically redistributed from three B-sites of the enlarged $2 \times 2$ unit cell of the monolayer to its fourth B-site, which becomes much more protruded than the three. This corresponds to the brighter spots on the p-sites which emerge with a period of $4.9 \AA$ on the observed STM image of the GIC surface..$^{5,6}$ At the same time the other three B-sites correspond to the $\mathrm{p}^{\prime}$ - and $\mathrm{p}^{\prime \prime}$-spots of lower intensity also observed in refs 5 and 6 . The external field, however, does not affect the LDOS on either A-site in the enlarged unit cell, and they remain weakly protruded on the STM images, in fair agreement with experiment. We see that in our approach we reproduce satisfactorily the localizing effect of the external field of the metal cations on the LDOS, which was recently mentioned in ref 14. However, our approach allows the reproduction in a consistent manner of also the inherent periodicity of $2.46 \AA$ related to the intrinsic features of the electronic structure of the graphite monolayer not sensitive to its environment. These features are ultimately controlled by the Coulomb interactions between electrons occupying the adjacent carbon sites, which were neglected in ref 14 , as were the terms describing the electron hopping between the site, the crucial terms in any description of electronic structure of extended systems. ${ }^{19}$

Finally we considered what effect the formation of a lowsymmetry (ordered) state could have on the observed STM image of GIC provided the ordering is due to the interactions between electrons located in the vicinity of the two segments of the Fermi surface of the monolayer. It turned out that the resulting pattern can have a modulation period remarkably close to the lower bound of the observed Moiré patterns. ${ }^{23}$ However, the general situation with the latter is not clear, since some strong indications supporting their artificial origin due to the STM sampling procedure were given recently. ${ }^{23}$ If, however, any evidence of the real existence of the Moiré patterns and precise measurements of their characteristics appear in the future, the purely electronic driving force of their formation proposed in the present paper can be reconsidered among others.

Acknowledgment. This work was supported by the Russian Foundation for Basic Research (RFBR) under Grant No 9403-9902. The author thanks R. Hoffmann, J.-P. Malrieu, M.B. Lepetite, M. V. Basilevsky, M. A. Kozhushner, and I. A. Misurkin for their kind encouragement and support. 


\section{References and Notes}

(1) Binnig, G.; Fuchs, H.; Gerber, Ch.; Rohrer, H.; Stoll, E.; Tosatti, E. Europhys. Lett. 1986, 1, 31 .

(2) Parks, S. I.; Quate, C. F. Appl. Phys. Lett. 1986, 48, 112.

(3) Soler, A. M.; Baro, N.; Garcia, J. M.; Rohrer, H. Phys. Rev. Lett. 1986, 57,444 .

(4) Hansma, P. K. Bull. Am. Phys. Soc. 1985, 30, 251. Kuwabara, M.; Clarke, D. R.; Smith, D. A. Appl. Phys. Lett. 1990, 56, 2396.

(5) Kelty, S. P.; Lieber, C. M. J. Phys. Chem. 1989, 93, 5983.

(6) Kelty, S. P.; Lieber, C. M. Phys. Rev. B 1989, 40, 5856.

(7) Tomanek, D.; Louie, S. G. Phys. Rev. B 1988, 37, 8327.

(8) Tomanek, D.; Louie, S. G.; Mamin, H. J.; Abraham, D. W.; Thomson, R. E.; Clarke, J. Phys. Rev. B 1987, 35, 7790.

(9) Land, T. A.; Michely, T.; Behm, R. J.; Hemminger, J. C.; Comsa, G. Surf. Sci. 1992, 264, 261.

(10) Tchougréeff, A. L.; Hoffmann, R. J. Phys. Chem. 1992, 96, 8993.

(11) Misurkin, I. A.; Ovchinnikov, A. A. Teor. Eksp. Khim. 1968, 4, 3 [in Russian]. Misurkin, I. A.; Ovchinnikov, A. A. Theor. Chim. Acta 1969, 13, 115. Misurkin, I. A.; Ovchinnikov, A. A. Teor. Eksp. Khim. 1976, 12 , 291 [in Russian]; Theor. Exp. Chem. 1976, 12, 225 [in English].

(12) Tersoff, J. Phys. Rev. Lett. 1986, 57, 440.
(13) Marchand, D.; Fretigny, C.; Lecomte, N.; Lagues, M. Synth. Met. 1988, 23, 156.

(14) Whangbo, M.-H.; Liang, W.; Ren, J.; Magonov, S. N.; Wawkuschewski, A. J. Phys. Chem. 1994, 98, 7602.

(15) Brandt, N. B.; Chudinov, S. M.; Ponomarev, Ya. G. Semimetals, Graphite and its Compounds; Modern Problems in Condensed Matter Sciences, Vol. 20.1; North-Holland: Amsterdam, 1988.

(16) Ando, T.; Fouler, A. B.; Stern, F. Rev. Mod. Phys. 1982, 54, 437.

(17) Tersoff, J.; Hamann, D. R. Phys. Rev. B 1985, 31, 805.

(18) Economou, E. N. Green's Functions in Quantum Physics; SpringerVerlag: Berlin, New York, 1979.

(19) Hoffmann, R. Solids and Surfaces: A Chemist's View of Bonding in Extended Structures; VCH: New York, 1988.

(20) Grunes, L. A.; Gates, I. P.; Ritsko, J. J.; Mele, E. J.; DiVincenzo, D. P.; Preil, M. E.; Fisher, J. E. Phys. Rev. B 1983, 28, 6681.

(21) Gelmukhanov, F.; Agren, H. J. Phys. B 1995, 28, 3699.

(22) Mattuck, R. D. A Guide to Feynman Diagrams in the Many-Body Problem; McGraw-Hill: New York, 1976.

(23) Li, J. T.; Berndt, R.; Schneider, W.-D. Appl. Phys. Lett. 1995, 67, 921.

JP953299U 